\title{
SYNTHESIS AND QUANTITATIVE STRUCTURE ACTIVITY RELATIONSHIP (QSAR) STUDY OF NEW 3- ALLYL-5-SUBSTITUTED-3,4,5,6-TETRAHYDRO-2H-1,3,5- THIADIAZINE-2-THIONES OF A POTENTIAL ANTIMICROBIAL ACTIVITY
}

Alaa M. Hayallah, and Awwad A. Radwan*

Department of Pharmaceutical Organic Chemistry, Faculty of Pharmacy, Assiut University, Assiut-71527, Egypt

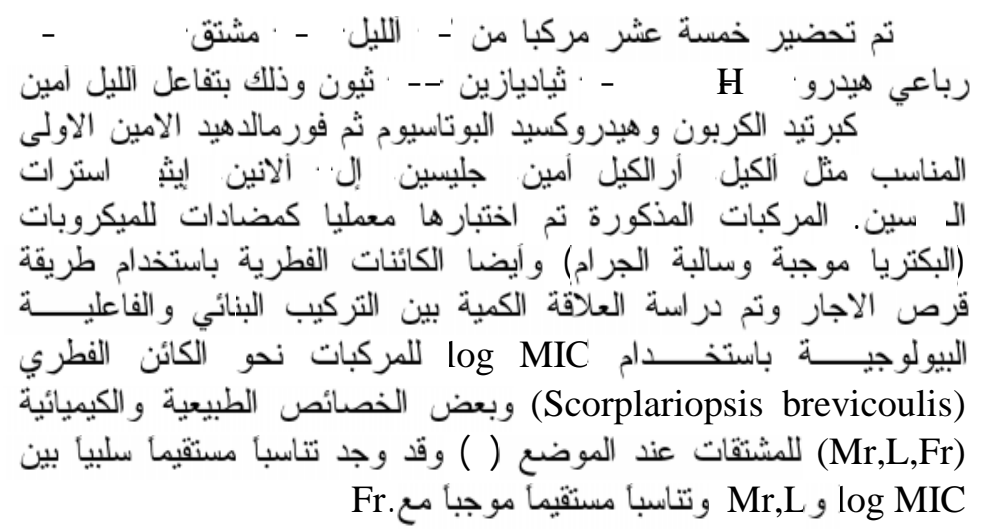

Fifteen new 3-allyl-5-substituted-3,4,5,6-tetrahydro-2H-1,3,5thiadiazine-2-thiones were synthesized by the reaction of allylamine with carbon disulfide and potassium hydroxide, followed by formaldehyde and appropriate primary amine such as alkyl, aralkylamines, glycine, L-alanine or ethyl glycine ester. The title compounds were tested, in vitro, for antimicrobial activity against gram-positive bacteria (Bacillus cereus, Staphylococcus aureus, Micrococcus leuteus), Gram-negative bacteria (Escherichia coli, Serratia marcescens, Pseudomonas aeruginosa), and some fungi (Aspergillus flavus, Aspergillus niger, candida albicans, Geotrichum candidum, Scopulariopsis brevicaulis and Trichophyton rubrum), using agar disc method. Quantitative structure activity relationship study was performed using the log form of MIC against Scopulariopsis brevicaulis fungi and some physicochemical descriptors $(M R, L$, and Fr) of substituents at N-5

Received in 19/11/2006, Received in revised form in 1/2/2007 \& Accepted in 11/2/2007

*Corresponding auther E-mail address: dhna_2001@hotmail.com. 
position. The log MIC were found to be negatively linearly correlated with MR and L and positively linearly correlated with Fr.

\section{INTRODUCTION}

Systemic mycosis in patients suffering from debilitating diseases such as neopliasis and in persons on long term total parentral nutrition ${ }^{1}$ is becoming critical for the need of more and better antifungal agents. Compounds carrying the tetrahydro1,3,5-thiadiazine-2-thione (THTT) skeleton have been reported to exhibit antibacterial $^{2-5}$, antifungal ${ }^{6-9}$ and antiviral properties ${ }^{10-13}$. The antimicrobial activities of THTT may be through the production of isothiocyanate and/ or dithiocarbamic acids ${ }^{3,14,15}$. These findings motivated our interest to design new derivatives of THTT as antimicrobial agents. In our effort to shed light on the structural requirements for the antifungal and antibacterial activities of the 1,3,5-thiadiazine-2-thione nucleus, we prepared several new derivatives. Study of structural requirements was based on fixing of lipophilic substituent (allyl group) at $\mathrm{N}-3$ position and incorporating different substituents at N-5 position ${ }^{15-17}$. The compounds were screened, and a variable antifungal and antibacterial activities were elaborated. Besides, we report classical quantitative structureactivity relationship studies (QSARs) using $\log$ MIC $(\mu \mathrm{M} / \mathrm{ml})$ against Scopulariopsis brevicaulis fungi and some electronic and steric descriptors of substituents at N-5 position. Results discussed below show optimum correlation when $\mathrm{Fr}, \mathrm{Mr}$ and $\mathrm{L}$ descriptors are combined together in one model. The statistics of the fitting are impressive and indicate that the equation (model) derived is good predictor of activity.

\section{EXPERIMENTAL}

\section{Materials and equipments}

Melting points were determined on an electrothermal melting point apparatus [Stuart Scientific, UK], and are uncorrected. Precoated silica gel plates (kiesel gel 0.25 mm, 60G F254, Merck) were used for thin layer chromatography. Developing solvent system of chloroform/methanol (10:3) was used and the spots were detected by ultraviolet light. IR spectra $(\mathrm{KBr}$ disc) were recorded on IR-470 Shimadzu spectrometer, Japan. ${ }^{1} \mathrm{H}-$ NMR Spectra were scanned on a Varian EM-360 L NMR spectrometer $(60 \mathrm{MHz})$ USA at Faculty of Pharmacy Assiut University. Chemical shifts are expressed in $\delta$ values (ppm) relative to TMS as an internal standard, using $\mathrm{CDCl}_{3}$ as a solvent. Elemental analyses were performed at the Department of Chemistry, Faculty of Science, Assiut University, Assiut, Egypt. Antimicrobial activity was performed at Mycology center AUMC, Department 
of Botany, Faculty of Science, Assiut University, Assiut, Egypt.

General procedure for synthesis of 3-allyl-5-substituted tetrahydro2H-1,3,5-thiadiazine-2-thiones; (4ao) $)^{4,5}$

Carbon disulfide $(60 \mathrm{mmol}, 3.6$ $\mathrm{ml}$ ) was added portionwise to a stirred mixture of the allylamine $\mathbf{1}(10 \mathrm{mmol}$, $0.75 \mathrm{ml}$ ) and potassium hydroxide (40\%, $20 \mathrm{mmol}, 1.4 \mathrm{ml})$. The reaction mixture was stirred for $3 \mathrm{~h}$. at ambient temperature. To the reaction mixture, which contains the potassium dithiocarbamates $\mathbf{2}$, formaldehyde solution $(35 \%, 22 \mathrm{mmol}, 1.63 \mathrm{ml})$, was added and the stirring was continued for further $1 \mathrm{~h}$. The resulting clear solution of $\mathbf{3}$ was added portionwise during $15 \mathrm{~min}$ to a stirred solution of the appropriate alkylamine, aralkylamine, glycine, Lalanine or ethyl glycine ester (10 mmol) in phosphate buffer $(\mathrm{pH} 7.8$, $20 \mathrm{ml}$ ). After stirring for $6 \mathrm{~h}$. at ambient temperature, the reaction mixture was acidified with dilute hydrochloric acid $(5 \%, \sim 15-18 \mathrm{ml})$ to $\mathrm{pH} 2$ and the stirring was continued for further $30 \mathrm{~min}$. The formed precipitate was collected by filtration, washed with $0.5 \%$ hydrochloric acid and dried. The crude solid was crystallized from methanol-chloroform (1:1) to afford 4a-o Yields, physical and spectral data are given in Tables I and II.

\section{Antimicrobial Activity \\ Organisms, culture conditions, and antimicrobial activity}

Six pathogenic fungal species were used in the present study: Aspergillus flavus, Aspergillus niger, candida albicans, Geotrichum candidum, Scopulariopsis brevicaulis and Trichophyton rubrum (Robin) Berkhout (a cause of candidiasis), Candida albicans and Trichophyton rubrum were isolated from clinical cases in the Assiut University hospitals. For antibacterial activity three gram-positive bacteria (Bacillus cereus, staphylococcus aureus, Micrococcus leuteus), and three gramnegative bacteria (Escherichia coli, Serratia marcescens, pseudomonas aeruginosa) were used. Spore suspension in sterile distilled water was prepared from 3-5 days old culture of the test fungi growing on SAD medium. The final spore concentration was $5 \times 104$ spores $/ \mathrm{ml}$. About $20 \mathrm{ml}$ of growth medium was introduced on sterilized plates of 9 $\mathrm{Cm}$ diameter and inoculated with $1 \mathrm{ml}$ of spore suspension. Plates were shaken gently to homogenize the inoculum. Antifungal activity of the tested compounds was performed by the standard agar disk diffusion method18 as follow: Sterile $6 \mathrm{~mm}$ filter paper disks (Whatman) were impregnated with $5 \mu \mathrm{l}$ solutions of the tested compounds $(10 \mathrm{mg} / \mathrm{ml}$ in DMSO). In addition, other disks were impregnated with the solvent (DMSO) and served as control. The impregnated disks were then dried for 1 hour and placed in the center of 
each plate. The seeded plates were incubated at $30 \pm 2{ }^{\circ} \mathrm{C}$ for 7 days. The radius of the inhibition zones (in $\mathrm{mm}$ ) were measured at successive intervals during the incubation period. Triplicate set were applied for each treatment. Results are given in Table III in comparison to reference drugs (clotrimazole and chloramphenicol) as antifungal and antimicrobial respectively.
For all compounds except the weak active compounds $\mathbf{4 l}, \mathbf{4 n}$, and 4o, the tube dilution method was used for determination of minimal inhibitory concentrations (MIC). The concentrations of the compounds in tubes were $1,1 / 2$ and $1 / 4 \mu \mathrm{mole} / \mathrm{ml}$, respectively ${ }^{7}$.

Table 1: Physicochemical data of the newly synthesized derivatives.

\begin{tabular}{|c|c|c|c|c|c|c|c|c|}
\hline \multirow{2}{*}{$\begin{array}{l}\text { Compd. } \\
\text { No }\end{array}$} & \multirow{2}{*}{$\mathrm{R}$} & \multirow{2}{*}{$\begin{array}{c}\text { Yield } \\
\%\end{array}$} & \multirow{2}{*}{ Formula } & \multirow{2}{*}{$\begin{array}{l}\text { M.p. } \\
{ }^{\circ} \mathrm{C}\end{array}$} & \multicolumn{4}{|c|}{ Elemental analysis (Calc/found) } \\
\hline & & & & & $\mathrm{C}$ & $\mathrm{H}$ & $\mathrm{N}$ & $\mathrm{S}$ \\
\hline $4 \mathbf{a}$ & $\mathrm{CH}_{3}$ & 45 & $\mathrm{C}_{7} \mathrm{H}_{12} \mathrm{~N}_{2} \mathrm{~S}_{2}$ & $61-3$ & $\begin{array}{l}44.65 \\
44.50\end{array}$ & $\begin{array}{l}6.42 \\
6.23\end{array}$ & $\begin{array}{l}14.88 \\
14.89\end{array}$ & \\
\hline $4 b$ & $\mathrm{C}_{2} \mathrm{H}_{5}$ & 60 & $\mathrm{C}_{8} \mathrm{H}_{14} \mathrm{~N}_{2} \mathrm{~S}_{2}$ & $48-50$ & $\begin{array}{l}47.49 \\
48.24 \\
\end{array}$ & $\begin{array}{l}6.97 \\
7.05 \\
\end{array}$ & $\begin{array}{l}13.84 \\
14.23 \\
\end{array}$ & \\
\hline $4 c$ & $\mathrm{n}-\mathrm{C}_{3} \mathrm{H}_{7}$ & 57 & $\begin{array}{l}\mathrm{C}_{9} \mathrm{H}_{16} \mathrm{~N}_{2} \mathrm{~S}_{2} \\
.1 / 2 \mathrm{H}_{2} \mathrm{O} \\
\end{array}$ & $36-8$ & $\begin{array}{l}48.00 \\
47.23 \\
\end{array}$ & $\begin{array}{l}7.56 \\
7.08 \\
\end{array}$ & $\begin{array}{l}12.44 \\
12.15 \\
\end{array}$ & $\begin{array}{l}28.44 \\
27.86 \\
\end{array}$ \\
\hline $4 d$ & $i-\mathrm{C}_{3} \mathrm{H}_{7}$ & 65 & $\mathrm{C}_{9} \mathrm{H}_{16} \mathrm{~N}_{2} \mathrm{~S}_{2}$ & $44-6$ & $\begin{array}{l}49.96 \\
50.45 \\
\end{array}$ & $\begin{array}{l}7.45 \\
6.92 \\
\end{array}$ & $\begin{array}{l}12.95 \\
13.09\end{array}$ & $\begin{array}{l}29.64 \\
29.12 \\
\end{array}$ \\
\hline $4 e$ & $\mathrm{n}-\mathrm{C}_{4} \mathrm{H}_{9}$ & 62 & $\mathrm{C}_{10} \mathrm{H}_{18} \mathrm{~N}_{2} \mathrm{~S}_{2}$ & $43-4$ & & & $\begin{array}{l}12.16 \\
12.07 \\
\end{array}$ & $\begin{array}{l}27.84 \\
27.27\end{array}$ \\
\hline $4 f$ & i-butyl & 60 & $\mathrm{C}_{10} \mathrm{H}_{18} \mathrm{~N}_{2} \mathrm{~S}_{2}$ & liquid & & & & \\
\hline $4 \mathrm{~g}$ & $\mathrm{t}-\mathrm{C}_{4} \mathrm{H}_{9}$ & 55 & $\mathrm{C}_{10} \mathrm{H}_{18} \mathrm{~N}_{2} \mathrm{~S}_{2}$ & $99-101$ & $\begin{array}{l}52.13 \\
51.32 \\
\end{array}$ & $\begin{array}{l}7.87 \\
7.51 \\
\end{array}$ & $\begin{array}{l}12.16 \\
12.02 \\
\end{array}$ & \\
\hline $4 h$ & $\mathrm{n}-\mathrm{C}_{5} \mathrm{H}_{11}$ & 70 & $\begin{array}{l}\mathrm{C}_{11} \mathrm{H}_{20} \mathrm{~N}_{2} \mathrm{~S}_{2} \\
2 \mathrm{H}_{2} \mathrm{O}\end{array}$ & $52-4$ & & & $\begin{array}{l}9.99 \\
9.94 \\
\end{array}$ & $\begin{array}{l}22.87 \\
22.14 \\
\end{array}$ \\
\hline $4 i$ & $\mathrm{n}-\mathrm{C}_{6} \mathrm{H}_{13}$ & 70 & $\begin{array}{l}\mathrm{C}_{12} \mathrm{H}_{22} \mathrm{~N}_{2} \mathrm{~S}_{2} .1 / 2 \\
\mathrm{H}_{2} \mathrm{O}\end{array}$ & $56-8$ & & & $\begin{array}{l}10.48 \\
10.78 \\
\end{array}$ & $\begin{array}{l}23.97 \\
24.04 \\
\end{array}$ \\
\hline $4 j$ & $\mathrm{CH}_{2} \mathrm{COOH}$ & 65 & $\begin{array}{l}\mathrm{C}_{8} \mathrm{H}_{12} \mathrm{~N}_{2} \mathrm{O}_{2} \mathrm{~S}_{2} \\
1 / 2 \mathrm{H}_{2} \mathrm{O}\end{array}$ & $132-4$ & $\begin{array}{l}39.83 \\
40.08 \\
\end{array}$ & $\begin{array}{l}5.39 \\
4.91 \\
\end{array}$ & $\begin{array}{l}11.62 \\
11.78 \\
\end{array}$ & $\begin{array}{l}26.55 \\
26.17 \\
\end{array}$ \\
\hline $4 \mathbf{k}$ & $\mathrm{CH}\left(\mathrm{CH}_{3}\right) \mathrm{COOH}$ & 60 & $\mathrm{C}_{9} \mathrm{H}_{14} \mathrm{~N}_{2} \mathrm{O}_{2} \mathrm{~S}_{2}$ & $111-13$ & $\begin{array}{l}43.88 \\
43.37 \\
\end{array}$ & $\begin{array}{l}5.73 \\
5.55 \\
\end{array}$ & $\begin{array}{l}11.37 \\
11.25 \\
\end{array}$ & $\begin{array}{l}26.03 \\
25.91 \\
\end{array}$ \\
\hline 41 & $\mathrm{CH}_{2} \mathrm{COOC}_{2} \mathrm{H}_{5}$ & 45 & $\mathrm{C}_{10} \mathrm{H}_{16} \mathrm{~N}_{2} \mathrm{O}_{2} \mathrm{~S}_{2}$ & $100-02$ & $\begin{array}{l}46.13 \\
45.12 \\
\end{array}$ & $\begin{array}{l}6.19 \\
6.04 \\
\end{array}$ & $\begin{array}{l}10.76 \\
10.73 \\
\end{array}$ & $\begin{array}{l}24.63 \\
24.69 \\
\end{array}$ \\
\hline $4 m$ & $\mathrm{CH}_{2}=\mathrm{CHCH}_{2}$ & 52 & $\mathrm{C}_{9} \mathrm{H}_{14} \mathrm{~N}_{2} \mathrm{~S}_{2}$ & $54-6$ & $\begin{array}{l}50.43 \\
50.86 \\
\end{array}$ & $\begin{array}{l}6.58 \\
6.66 \\
\end{array}$ & $\begin{array}{l}13.07 \\
13.31 \\
\end{array}$ & \\
\hline $4 n$ & $\mathrm{C}_{6} \mathrm{H}_{5}-\mathrm{CH}_{2}$ & 55 & $\mathrm{C}_{13} \mathrm{H}_{16} \mathrm{~N}_{2} \mathrm{~S}_{2}$ & $51-3$ & $\begin{array}{l}59.05 \\
59.23 \\
\end{array}$ & $\begin{array}{l}6.10 \\
6.05 \\
\end{array}$ & $\begin{array}{l}10.59 \\
10.53 \\
\end{array}$ & $\begin{array}{l}24.25 \\
23.16 \\
\end{array}$ \\
\hline 40 & $\mathrm{C}_{6} \mathrm{H}_{5}-\mathrm{CH}\left(\mathrm{CH}_{3}\right)$ & 87 & $\mathrm{C}_{14} \mathrm{H}_{18} \mathrm{~N}_{2} \mathrm{~S}_{2}$ & $118-20$ & $\begin{array}{l}60.39 \\
59.94\end{array}$ & $\begin{array}{l}6.52 \\
6.47\end{array}$ & $\begin{array}{l}10.06 \\
10.07\end{array}$ & $\begin{array}{l}23.03 \\
23.33\end{array}$ \\
\hline
\end{tabular}


Table II: The ${ }^{1} \mathrm{H}-\mathrm{NMR}$ chemical shifts of synthesized compounds in $\mathrm{CDCl}_{3}$

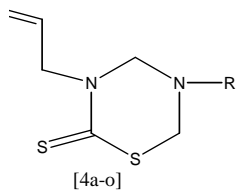

\begin{tabular}{|c|c|c|c|c|}
\hline No & R & Allyl-N-3 & $\mathrm{CH}_{2}$ at $\mathrm{C} 4$ and $\mathrm{C} 6$ & R-N-5 \\
\hline $4 a$ & $\mathrm{CH}_{3}$ & $\begin{array}{l}4.8\left(\mathrm{~d}, 2 \mathrm{H}, \mathrm{CH}_{2}-\mathrm{N}\right), 5.3(\mathrm{~d}, 1 \mathrm{H}, \\
\left.=\mathrm{CH}_{2}\right), 5.6\left(\mathrm{~d}, 1 \mathrm{H},=\mathrm{CH}_{2}\right), 6.4- \\
5.8(\mathrm{~m}, 1 \mathrm{H},=\mathrm{CH})\end{array}$ & $\begin{array}{l}.5(\mathrm{~s}, 2 \mathrm{H}) \\
4.6(\mathrm{~s}, 2 \mathrm{H})\end{array}$ & $2.7\left(\mathrm{~s}, 3 \mathrm{H}, \mathrm{CH}_{3}\right)$ \\
\hline $4 b$ & $\mathrm{C}_{2} \mathrm{H}_{5}$ & $\begin{array}{l}4.8\left(\mathrm{~d}, 2 \mathrm{H}, \mathrm{CH}_{2}-\mathrm{N}\right), 5.3(\mathrm{~d}, 1 \mathrm{H}, \\
\left.=\mathrm{CH}_{2}\right), 5.5\left(\mathrm{~d}, 1 \mathrm{H},=\mathrm{CH}_{2}\right), 6.4- \\
5.7(\mathrm{~m}, 1 \mathrm{H},=\mathrm{CH})\end{array}$ & $\begin{array}{l}4.5(\mathrm{~s}, 2 \mathrm{H}) \\
4.6(\mathrm{~s}, 2 \mathrm{H})\end{array}$ & $\begin{array}{l}1.2\left(\mathrm{t}, 3 \mathrm{H}, \mathrm{CH}_{2} \mathrm{C}_{3}\right), \\
2.9\left(\mathrm{q}, 2 \mathrm{H}, \mathrm{CH}_{2} \mathrm{CH}_{3}\right)\end{array}$ \\
\hline $4 c$ & $\mathrm{n}-\mathrm{C}_{3} \mathrm{H}_{7}$ & $\begin{array}{l}4.8\left(\mathrm{~d}, 2 \mathrm{H}, \mathrm{CH}_{2}-\mathrm{N}\right), 5.3(\mathrm{~d}, 1 \mathrm{H}, \\
\left.=\mathrm{CH}_{2}\right), 5.5\left(\mathrm{~d}, 1 \mathrm{H},=\mathrm{CH}_{2}\right), 6.4- \\
5.7(\mathrm{~m}, 1 \mathrm{H},=\mathrm{CH})\end{array}$ & $\begin{array}{l}4.4(\mathrm{~s}, 2 \mathrm{H}) \\
4.6(\mathrm{~s}, 2 \mathrm{H})\end{array}$ & $\begin{array}{l}1.0\left(\mathrm{t}, 3 \mathrm{H}, \mathrm{CH}_{3}\right), 1.9-1.3(\mathrm{~m}, \\
\left.2 \mathrm{H}, \quad \mathrm{CH}_{2} \mathrm{CH}_{2}\right), 2.8 \quad(\mathrm{t}, 2 \mathrm{H}, \\
\left.\mathrm{CH}_{2} \mathrm{CH}_{2}\right)\end{array}$ \\
\hline $4 d$ & $i-\mathrm{C}_{3} \mathrm{H}_{7}$ & $\begin{array}{l}5.1\left(\mathrm{~d}, 2 \mathrm{H}, \mathrm{CH}_{2}-\mathrm{N}\right), 5.6(\mathrm{~d}, 1 \mathrm{H}, \\
\left.=\mathrm{CH}_{2}\right), 5.8\left(\mathrm{~d}, 1 \mathrm{H},=\mathrm{CH}_{2}\right), 6.8- \\
6.0(\mathrm{~m}, 1 \mathrm{H},=\mathrm{CH})\end{array}$ & $\begin{array}{l}4.7(\mathrm{~s}, 2 \mathrm{H}) \\
4.8(\mathrm{~s}, 2 \mathrm{H}\end{array}$ & $\begin{array}{l}1.3\left(\mathrm{~d}, 6 \mathrm{H}, 2\left(\mathrm{C}^{3}\right), 3.7-3.2(\mathrm{~m},\right. \\
1 \mathrm{H}, \mathrm{C} \underline{\mathrm{H}})\end{array}$ \\
\hline $4 e$ & $\mathrm{n}-\mathrm{C}_{4} \mathrm{H}_{9}$ & $\begin{array}{l}4.8\left(\mathrm{~d}, 2 \mathrm{H}, \mathrm{CH}_{2}-\mathrm{N}\right), 5.3(\mathrm{~d}, 1 \mathrm{H}, \\
\left.=\mathrm{CH}_{2}\right), 5.5\left(\mathrm{~d}, 1 \mathrm{H},=\mathrm{CH}_{2}\right) 6.7- \\
5.8(\mathrm{~m}, 1 \mathrm{H},=\mathrm{CH})\end{array}$ & $\begin{array}{l}4.5(\mathrm{~s}, 2 \mathrm{H}) \\
4.5(\mathrm{~s}, 2 \mathrm{H})\end{array}$ & $\begin{array}{l}\left.1.00 \quad \text { (t, } 3 \mathrm{H}, \quad \mathrm{CH}_{3}\right), \quad 2.4-1.1 \\
\left.\text { (br.m,4H, } \quad \mathrm{CH}_{2} \mathrm{CH}_{2} \mathrm{CH}_{2} \mathrm{CH}_{3}\right), \\
\left.2.9 \text { (t, } 2 \mathrm{H}, \mathrm{N5} 5-\mathrm{CH}_{2}\right)\end{array}$ \\
\hline $4 f$ & i- $\mathrm{C}_{4} \mathrm{H}_{9}$ & $\begin{array}{l}5.1\left(\mathrm{~d}, 2 \mathrm{H}, \mathrm{CH}_{2}-\mathrm{N}\right) \\
5.6\left(\mathrm{~d}, 1 \mathrm{H},=\mathrm{CH}_{2}\right) \\
5.8\left(\mathrm{~d}, 1 \mathrm{H},=\mathrm{CH}_{2}\right) \\
6.8-5.1(\mathrm{~m}, 1 \mathrm{H},=\mathrm{CH})\end{array}$ & $\begin{array}{l}4.7(\mathrm{~s}, 2 \mathrm{H}) \\
4.8(\mathrm{~s}, 2 \mathrm{H})\end{array}$ & $\begin{array}{l}1.0\left(\mathrm{~d}, 6 \mathrm{H}, 2 \mathrm{CH}_{3}\right), 2.3-1.4 \\
(\mathrm{~m}, 1 \mathrm{H}, \mathrm{CH}), 2.8(\mathrm{~d}, 2 \mathrm{H}, \mathrm{N} 5- \\
\mathrm{CH}_{2)}\end{array}$ \\
\hline $4 g$ & $\mathrm{t}-\mathrm{C}_{4} \mathrm{H}_{9}$ & $\begin{array}{l}4.8\left(\mathrm{~d}, 2 \mathrm{H}, \mathrm{CH}_{2}-\mathrm{N}\right), 5.3(\mathrm{~d}, 1 \mathrm{H}, \\
\left.=\mathrm{CH}_{2}\right), 5.5\left(\mathrm{~d}, 1 \mathrm{H},=\mathrm{CH}_{2}\right), 6.4- \\
5.8(\mathrm{~m}, 1 \mathrm{H},=\mathrm{CH})\end{array}$ & $\begin{array}{l}4.6(\mathrm{~s}, 2 \mathrm{H}) \\
4.7(\mathrm{~s}, 2 \mathrm{H})\end{array}$ & $1.3\left(\mathrm{~s}, 9 \mathrm{H}, 3 \mathrm{CH}_{3}\right)$ \\
\hline $4 h$ & n- $\mathrm{C}_{5} \mathrm{H}_{11}$ & $\begin{array}{l}4.8\left(\mathrm{~d}, 2 \mathrm{H}, \mathrm{CH}_{2}-\mathrm{N}\right), 5.3(\mathrm{~d}, 1 \mathrm{H}, \\
\left.=\mathrm{CH}_{2}\right), 5.5\left(\mathrm{~d}, 1 \mathrm{H},=\mathrm{CH}_{2}\right), 6.4- \\
5.7(\mathrm{~m}, 1 \mathrm{H},=\mathrm{CH})\end{array}$ & $\begin{array}{l}4.4(\mathrm{~s}, 2 \mathrm{H}) \\
4.5(\mathrm{~s}, 2 \mathrm{H})\end{array}$ & $\begin{array}{l}0.9\left(\mathrm{t}, 3 \mathrm{H}, \mathrm{CH}_{3}\right), 1.9-1.1(\mathrm{~m}, \\
\left.6 \mathrm{H}, 3 \mathrm{CH}_{2}\right), 2.8(\mathrm{t}, 2 \mathrm{H}, \mathrm{N} 5- \\
\left.\mathrm{CH}_{2}\right)\end{array}$ \\
\hline $4 \mathbf{i}$ & $\mathrm{n}-\mathrm{C}_{6} \mathrm{H}_{13}$ & $\begin{array}{l}4.8\left(\mathrm{~d}, 2 \mathrm{H}, \mathrm{CH}_{2}-\mathrm{N}\right), 5.3(\mathrm{~d}, 1 \mathrm{H}, \\
\left.=\mathrm{CH}_{2}\right), 5.5\left(\mathrm{~d}, 1 \mathrm{H},=\mathrm{CH}_{2}\right), 6.4- \\
5.7(\mathrm{~m}, 1 \mathrm{H},=\mathrm{CH}\end{array}$ & $\begin{array}{l}4.4(\mathrm{~s}, 2 \mathrm{H}) \\
4.5(\mathrm{~s}, 2 \mathrm{H})\end{array}$ & $\begin{array}{l}0.9\left(\mathrm{t}, 3 \mathrm{H}, \mathrm{CH}_{3}\right), 1.9-1.1(\mathrm{~m}, \\
\left.6 \mathrm{H}, 4 \mathrm{CH}_{2}\right), 2.8(\mathrm{t}, 2 \mathrm{H}, \mathrm{N} 5- \\
\left.\mathrm{CH}_{2}\right)\end{array}$ \\
\hline $4 j$ & $\mathrm{CH}_{2} \mathrm{COOH}$ & $\begin{array}{l}4.8\left(\mathrm{~d}, 2 \mathrm{H}, \mathrm{CH}_{2}-\mathrm{N}\right), 5.3(\mathrm{~d}, 1 \mathrm{H}, \\
\left.=\mathrm{CH}_{2}\right), 5.5\left(\mathrm{~d}, 1 \mathrm{H},=\mathrm{CH}_{2}\right) \\
6.4-5.8(\mathrm{~m}, 1 \mathrm{H},=\mathrm{CH})\end{array}$ & $\begin{array}{l}4.5(\mathrm{~s}, 2 \mathrm{H}) \\
4.6(\mathrm{~s}, 2 \mathrm{H})\end{array}$ & $\begin{array}{l}\left.3.7 \text { (s, } 2 \mathrm{H}, \mathrm{C} \underline{\mathrm{H}}_{2} \mathrm{COOH}\right) \\
12 \text { (br. s, } 1 \mathrm{H}, \text { exch. } \mathrm{COO} \underline{\mathrm{H}})\end{array}$ \\
\hline $4 k$ & $(-) \mathrm{CH}\left(\mathrm{CH}_{3}\right) \mathrm{COOH}$ & $\begin{array}{l}4.9\left(\mathrm{~d}, 2 \mathrm{H}, \mathrm{CH}_{2}-\mathrm{N}\right), 5.3(\mathrm{~d}, 1 \mathrm{H}, \\
\left.=\mathrm{CH}_{2}\right), 5.6\left(\mathrm{~d}, 1 \mathrm{H},=\mathrm{CH}_{2}\right), 6.5- \\
5.8(\mathrm{~m}, 1 \mathrm{H},=\mathrm{CH})\end{array}$ & $4.7(\mathrm{~s}, 4 \mathrm{H})$ & $\begin{array}{l}1.5\left(\mathrm{~d}, 3 \mathrm{H}, \mathrm{CH}_{3}\right), \\
4.0\left(\mathrm{q}, 2 \mathrm{H}, \mathrm{CHCH}_{3}\right), \\
10.9 \text { (br. s, } 1 \mathrm{H}, \text { exch. } \mathrm{COOH})\end{array}$ \\
\hline 41 & $\mathrm{CH}_{2} \mathrm{COOC}_{2} \mathrm{H}_{5}$ & $\begin{array}{l}4.8\left(\mathrm{~d}, 2 \mathrm{H}, \mathrm{CH}_{2}-\mathrm{N}\right), 5.3(\mathrm{~d}, 1 \mathrm{H}, \\
\left.=\mathrm{CH}_{2}\right), 5.5\left(\mathrm{~d}, 1 \mathrm{H},=\mathrm{CH}_{2}\right), 6.4- \\
5.8(\mathrm{~m}, 1 \mathrm{H},=\mathrm{CH})\end{array}$ & $\begin{array}{l}4.5(\mathrm{~s}, 2 \mathrm{H}) \\
4.6(\mathrm{~s}, 2 \mathrm{H})\end{array}$ & $\begin{array}{l}4.3\left(\mathrm{q}, 2 \mathrm{H}, \mathrm{COOCH}_{2} \mathrm{CH}_{3}\right) \\
3.7\left(\mathrm{~s}, 2 \mathrm{H}, \mathrm{CH}_{2} \mathrm{COOC}_{2} \mathrm{H}_{5}\right) \\
1.3\left(\mathrm{t}, 3 \mathrm{H}, \mathrm{COOCH}_{2} \mathrm{CH}_{3}\right)\end{array}$ \\
\hline $4 m$ & $\mathrm{CH}_{2}=\mathrm{CHCH}_{2}$ & $\begin{array}{l}4.8\left(\mathrm{~d}, 2 \mathrm{H}, \mathrm{CH}_{2}-\mathrm{N}\right), 5.3(\mathrm{~d}, 1 \mathrm{H} \text { of } \\
\left.=\mathrm{CH}_{2}\right), 5.5\left(\mathrm{~d}, 1 \mathrm{H} \text { of }=\mathrm{CH}_{2}\right), 6.5- \\
5.7(\mathrm{~m}, 1 \mathrm{H} \text { of }=\mathrm{CH})\end{array}$ & $\begin{array}{l}4.5(\mathrm{~s}, 2 \mathrm{H}) \\
4.6(\mathrm{~s}, 2 \mathrm{H})\end{array}$ & $\begin{array}{l}3.5\left(\mathrm{~d}, 2 \mathrm{H}, \mathrm{N} 5-\mathrm{CH}_{2}\right), 5.3(\mathrm{~d}, \\
\left.1 \mathrm{H} \text { of }=\mathrm{CH}_{2}\right), 5.5(\mathrm{~d}, 1 \mathrm{H} \text { of } \\
\left.=\mathrm{CH}_{2}\right), 6.5-5.7(\mathrm{~m}, 1 \mathrm{H} \text { of }=\mathrm{CH})\end{array}$ \\
\hline $4 n$ & $\mathrm{C}_{6} \mathrm{H}_{5}-\mathrm{CH}_{2}$ & $\begin{array}{l}4.7\left(\mathrm{~d}, 2 \mathrm{H}, \mathrm{CH}_{2}-\mathrm{N}\right), 5.3(\mathrm{~d}, 1 \mathrm{H}, \\
\left.=\mathrm{CH}_{2}\right), 5.4\left(\mathrm{~d}, 1 \mathrm{H},=\mathrm{CH}_{2}\right), 6.4- \\
5.7(\mathrm{~m}, 1 \mathrm{H},=\mathrm{CH})\end{array}$ & $4.5(\mathrm{~s}, 4 \mathrm{H})$ & $\begin{array}{l}7.5\left(\mathrm{~s}, 5 \mathrm{H}, \mathrm{C}_{6} \underline{\mathrm{H}}_{5}-\mathrm{CH}_{2}\right) \\
4.1\left(\mathrm{~s}, 2 \mathrm{H}, \mathrm{C}_{6} \mathrm{H}_{5}-\mathrm{CH}_{2}\right)\end{array}$ \\
\hline 40 & ( $\pm \mathrm{C}_{6} \mathrm{H}_{5}-\mathrm{H}\left(\mathrm{CH}_{3}\right)$ & $\begin{array}{l}5.1\left(\mathrm{~d}, 2 \mathrm{H}, \mathrm{CH}_{2}-\mathrm{N}\right), 5.4(\mathrm{~d}, 1 \mathrm{H}, \\
\left.=\mathrm{CH}_{2}\right), 5.7\left(\mathrm{~d}, 1 \mathrm{H},=\mathrm{CH}_{2}\right), 6.7- \\
6.1(\mathrm{~m}, 1 \mathrm{H},=\mathrm{CH})\end{array}$ & $\begin{array}{l}4.5-4.3(\mathrm{~m}, 5 \mathrm{H}, \\
\left.2 \mathrm{CH}_{2} \text { and } \mathrm{N} 5-\mathrm{CH}\right)\end{array}$ & $\begin{array}{l}1.6\left(\mathrm{~d}, 3 \mathrm{H}, \mathrm{C}_{6} \mathrm{H}_{5} \mathrm{CH}\left(\mathrm{CH}_{3}\right), 7.9\right. \\
\left(\mathrm{s}, 5 \mathrm{H}, \mathrm{C}_{6} \underline{\mathrm{H}}_{5}\right),\end{array}$ \\
\hline
\end{tabular}


Table III: Antimicrobial activity of the tested compounds (expressed as the diameter of the inhibition zone ${ }^{a}$ ).

\begin{tabular}{|c|c|c|c|c|c|c|c|c|c|c|c|c|}
\hline No & 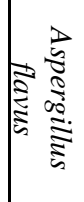 & 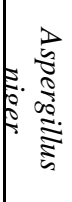 & 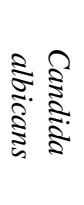 & 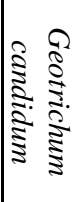 & 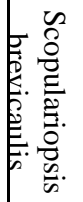 & 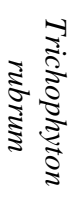 & ${ }_{\delta}^{\infty}$ & 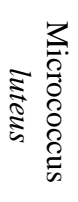 & 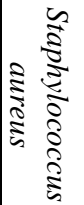 & 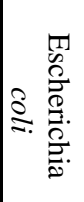 & 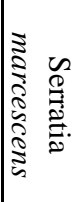 & 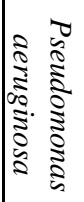 \\
\hline $4 a$ & 20 & 20 & 21 & 24 & 14 & 20 & 20 & 50 & 18 & 25 & 14 & - \\
\hline $4 b$ & 7 & 21 & 20 & 20 & 14 & 15 & 20 & 32 & 15 & 20 & 12 & 10 \\
\hline $4 c$ & 14 & 17 & 15 & 15 & 20 & 14 & 19 & 40 & 11 & 25 & 10 & - \\
\hline 4d & - & 16 & 17 & 18 & 16 & 12 & 20 & 30 & 16 & 20 & 6 & 10 \\
\hline $4 e$ & 15 & 20 & 14 & 12 & 15 & 13 & 15 & 35 & 10 & 15 & 10 & 13 \\
\hline $4 f$ & 13 & 20 & 13 & 12 & 13 & 11 & 15 & 30 & 12 & 20 & 10 & 7 \\
\hline $4 \mathrm{~g}$ & 12 & - & 13 & 14 & 12 & 11 & 15 & 20 & 13 & 20 & 15 & 10 \\
\hline $4 \mathrm{~h}$ & 11 & 11 & 11 & 10 & 11 & 11 & 11 & 15 & 10 & 13 & 7 & 7 \\
\hline$\overline{4 i}$ & - & 15 & 12 & 12 & 12 & - & 7 & 22 & 10 & 10 & 6 & - \\
\hline $4 \mathbf{j}$ & 13 & 20 & 13 & 20 & 13 & 14 & 18 & 50 & 17 & 26 & 10 & 7 \\
\hline $4 k$ & 20 & 22 & 15 & 12 & 20 & 15 & 20 & 26 & 15 & 22 & 16 & 12 \\
\hline 41 & - & - & - & - & - & - & 13 & 30 & 10 & 17 & 7 & - \\
\hline $4 m$ & - & 20 & 10 & 13 & 13 & 11 & 19 & 26 & 12 & 16 & 7 & - \\
\hline $4 n$ & - & - & 9 & - & - & - & - & 10 & 6 & 10 & - & - \\
\hline 40 & - & - & 6 & - & - & - & - & p.i. & - & - & - & - \\
\hline Ref.1 & - & - & - & - & - & - & 50 & 52 & 30 & 40 & - & 30 \\
\hline Ref.2 & 23 & 22 & 30 & 20 & 22 & 13 & - & - & - & - & - & - \\
\hline
\end{tabular}

- a) Average of three observations.

- Inhibition zone in $\mathrm{mm}$. Disc diameter is $6 \mathrm{~mm}$.

-“_“" no inhibition zone.

- Ref. 1 Chloramphenicol as antibacterial.

- Ref. 2 Clotrimazole as antifungal.

Quantitative structure activity relationship

\section{The inhibition values}

Quantitative structure-activity rela-tionship study (QSARs) was performed using physicochemical activity relationship methods $(\mathrm{PAR})^{19}$. The minimal inhibitor concentrations (MIC) in $\mu \mathrm{M} / \mathrm{ml}$ against Scopulariopsis brevicaulis fungi were adopted. These values were converted to their log form and used in the present investigations.

\section{The descriptors}

hydrophobic effect was treated by use of fragmental constants, $\mathrm{Fr}^{20}$, instead of Pi-value. The former was calculated by summing structural elements rather than substitution to calculate the $\log P$ value of an analog. Bulk substituent constants were employed using the molar refraction, 
$\mathrm{MR}^{21 \& 22}$. In addition, Sterimol-L values $^{23}$ (Steric length parameter) were measured along the substitution point bond axis.

\section{RESULTS AND DISCUSSION}

\section{Chemistry}

The target compounds were synthesized by the reaction of allylamine (1) with carbon disulphide and potassium hydroxide. The resulting dithiocarbamate potassium salt (2) without isolation was then allowed to react with formaldehyde to give (3) which was not isolated. The latter derivative was then allowed to react with the appropriate alkylamine, aralkylamine, glycine, L-alanine, or ethyl glycine ester in presence of phosphate buffer $(\mathrm{pH}=7.8)$ to give (4a-o) (see Scheme 1). It was suggested that the reaction proceeds through the formation of the intermediates (2) and (3) in situ ${ }^{3-6}$. Structures of the synthesized compounds were verified on the bases of spectral and elemental methods of analyses. Table 1, shows the physicochemical constants of the newly synthesized derivatives (4a-o). All spectral data are in accordance with the expected structures. The IR spectra of compounds 4a-o showed bands at 2840-2960 $\mathrm{cm}^{-1}$ (aliphatic C$\mathrm{H}$ stretching); 3030-3060 $\mathrm{cm}^{-1}$ (olefinic C-H stretching) and at about 1420-1455 $\mathrm{cm}^{-1} \quad(\mathrm{C}=\mathrm{S}$ stretching $)$. Moreover, compounds $\mathbf{4 j}$ and $\mathbf{4 k}$ showed the characteristic stretching absorption of the $\mathrm{OH}$ group at 2500-3200 $\mathrm{cm}^{-1}$ and $\mathrm{C}=\mathrm{O}$ at 1705 $1715 \mathrm{~cm}^{-1}$. Compound 41 showed the ester $\mathrm{C}=\mathrm{O}$ stretching at $1745 \mathrm{~cm}^{-1}$ and $\mathrm{C}-\mathrm{O}$ stretching very strong band at $1240 \mathrm{~cm}^{-1}$.

In the ${ }^{1} \mathrm{H}-\mathrm{NMR}$ spectra, a common pattern was found for the methylenes of $\mathrm{C} 4, \mathrm{C} 6$ and for allyl group at $\mathrm{N}-3[\delta$ 4.74-5.08 $(\mathrm{d}, 2 \mathrm{H}$, $\left.\mathrm{CH}_{2}-\mathrm{N}\right), 5.25-5.58\left(\mathrm{~d}, 1 \mathrm{H},=\mathrm{CH}_{2}\right)$, 5.43-5.83 $\left(\mathrm{d}, 1 \mathrm{H},=\mathrm{CH}_{2}\right), 5.7-6.4(\mathrm{~m}$, $1 \mathrm{H},=\mathrm{CH}-)$ in all derivatives. The characteristic differences in sets and patterns were attributed to the N-5side chain (Table II).

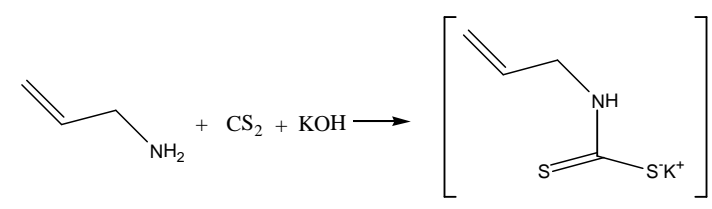

[1]

[2]

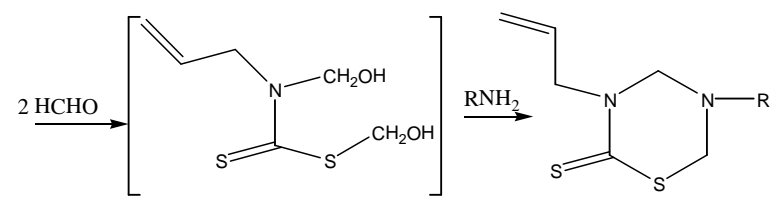

[3]

[4a-o]

Scheme 1: $\mathrm{R}=\mathrm{CH}_{3}(\mathrm{a}), \mathrm{C}_{2} \mathrm{H}_{5}$ (b), n- $\mathrm{C}_{3} \mathrm{H}_{7}(\mathrm{c}), \mathrm{i}-\mathrm{C}_{3} \mathrm{H}_{7}(\mathrm{~d}), \mathrm{n}-\mathrm{C}_{4} \mathrm{H}_{9}(\mathrm{e}), \mathrm{i}-\mathrm{C}_{4} \mathrm{H}_{9}(\mathrm{f})$, tert- $\mathrm{C}_{4} \mathrm{H}_{9}(\mathrm{~g}), \mathrm{n}$ $\mathrm{C}_{5} \mathrm{H}_{11}(\mathrm{~h}), \mathrm{C}_{6} \mathrm{H}_{13}(\mathrm{i}), \mathrm{CH}_{2} \mathrm{COOH}(\mathrm{j}), \quad \mathrm{CH}\left(\mathrm{CH}_{3}\right) \mathrm{COOH}(\mathrm{k}),-\mathrm{CH}_{2} \mathrm{COOC}_{2} \mathrm{H}_{5}$ (l), $\mathrm{CH}_{2}=\mathrm{CHCH}_{2}(\mathrm{~m}), \mathrm{C}_{6} \mathrm{H}_{5}-\mathrm{CH}_{2}(\mathrm{n}), \mathrm{C}_{6} \mathrm{H}_{5}-\mathrm{CH}\left(\mathrm{CH}_{3}\right)$ (o). 


\section{Antimicrobial Activity}

The synthesized compounds (4ao) were tested for their antifungal activity in vitro against Aspergillus flavus, Aspergillus niger, Candida albicans, Geotrichum candidum, Scopulariopsis brevicaulis and Trichophyton rubrum fungi using the standard agar diffusion method ${ }^{17}$ in comparison to clotrimazole as a standard drug. Table III shows the results of the antifungal activity of the tested compounds expressed as the inhibition zone in $\mathrm{mm}$. Also, the same compounds were tested, in vitro, for their antibacterial activity against gram-positive bacteria (Bacillus cereus, Staphylococcus aureus, Micrococcus leuteus), and gramnegative bacteria (Escherichia coli, Serratia marcescens, Pseudomonas aeruginosa) using chloramphenicol as a standard drug. The antimicrobial study explored variable activities according to variation of substituents at N-5 position of the THTT moiety. The results clearly indicate that the introduction of a variety of groups at N-5 gave good antifungal and moderate antibacterial compounds 4a-k and $\mathbf{4 m}$, relative to the reference clotrimazole or chloramphenicol respectively. However compounds 4l, $\mathbf{4 n}$, and 40 revealed no antifungal and weak antibacterial activity.

For all compounds except the weak active compounds $\mathbf{4 l}, \mathbf{4 n}$, and 4o, the tube dilution method was used for determination of minimal inhibitory concentrations (MIC) Table IV. The concentrations of the compounds in tubes were $1,1 / 2$ and $1 / 4$ $\mu \mathrm{M} / \mathrm{ml}$, respectively ${ }^{7}$.

Table IV: Antimicrobial activity of the tested compounds (expressed as the MIC in $\mu \mathrm{M} / \mathrm{ml})$.

\begin{tabular}{|c|c|c|c|c|c|c|c|c|c|c|c|c|}
\hline No & 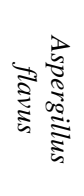 & 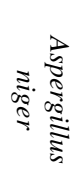 & 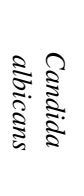 & 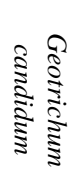 & 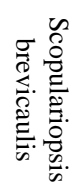 & 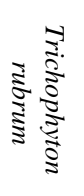 & 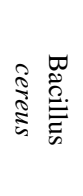 & 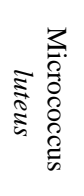 & 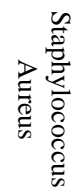 & 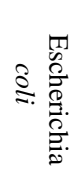 & 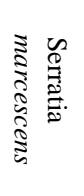 & 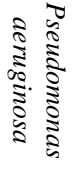 \\
\hline $4 a$ & 13.27 & 13.27 & 26.55 & 53.1 & 26.55 & 13.27 & 26.55 & 13.27 & 26.55 & - & 13.27 & - \\
\hline $4 b$ & 49.42 & 12.35 & 12.35 & 49.42 & 24.71 & 12.35 & 24.71 & 12.35 & 49.42 & 12.35 & - & 49.42 \\
\hline $4 c$ & 23.11 & 23.11 & 11.55 & - & 23.11 & 11.55 & 46.22 & - & 46.22 & 11.55 & - & - \\
\hline $4 d$ & - & 23.11 & 11.55 & 23.11 & 23.11 & 11.55 & 23.11 & 23.11 & 46.22 & 11.55 & 23.11 & - \\
\hline $4 e$ & 21.7 & 10.85 & 21.7 & 43.4 & 10.85 & 10.85 & $\begin{array}{l}43.4 \\
\end{array}$ & 10.85 & 43.4 & 10.85 & 10.85 & - \\
\hline 4f & 43.4 & 10.85 & 21.7 & 21.7 & 21.7 & 21.7 & 43.4 & 10.85 & 43.4 & 10.85 & - & - \\
\hline $4 \mathrm{~g}$ & 43.4 & - & 10.85 & 21.7 & 21.7 & 21.7 & 43.4 & 10.85 & 43.4 & 10.85 & 10.85 & - \\
\hline 4h & 40.91 & 40.91 & 40.91 & - & 10.23 & 10.23 & 40.91 & 10.23 & 40.91 & 10.23 & - & - \\
\hline $4 i$ & - & 38.68 & - & 19.34 & 9.67 & - & - & 9.67 & 38.68 & - & - & - \\
\hline $4 j$ & - & 10.76 & - & - & 10.76 & 10.76 & 21.5 & 10.76 & 21.5 & - & 10.76 & - \\
\hline $4 k$ & 10.16 & - & 10.16 & 40.64 & 10.16 & 10.16 & 40.46 & 10.16 & 40.46 & - & - & 10.16 \\
\hline $4 \mathrm{~m}$ & - & 11.66 & - & - & 23.32 & 23.32 & 46.65 & 11.66 & 46.65 & 11.66 & 11.66 & - \\
\hline
\end{tabular}


Quantitative structure activity relationship

The structural details of the synthesized compounds used in this investigation are given in scheme 1 . The antifungal activity against Scopulariopsis brevicaulis in $\log$ unit (log MIC) of this set are recorded in Table V. This table also contains the values of the descriptor parameters Fr, MR, and L. Results shown in Table VI proved weak correlation of the independent variables with each others and weak to moderate correlation with the dependent variable.

Table V: Dependent and independent values of the compounds.

\begin{tabular}{||c|c|c|c|c|}
\hline $\begin{array}{c}\text { Compd. } \\
\text { No. }\end{array}$ & $\begin{array}{c}\text { Log } \\
\mathrm{MIC}\end{array}$ & $\mathrm{Fr}^{*}$ & $\mathrm{MR}^{* *}$ & $\mathrm{~L}^{* *}$ \\
\hline $\mathbf{4 a}$ & 1.42 & 0.70 & 5.65 & 0.30 \\
\hline $\mathbf{4 b}$ & 1.39 & 1.40 & 10.30 & 4.11 \\
\hline $\mathbf{4 c}$ & 1.36 & 1.93 & 14.96 & 5.05 \\
\hline $\mathbf{4 d}$ & 1.36 & 1.63 & 14.96 & 4.11 \\
\hline $\mathbf{4 e}$ & 1.03 & 2.46 & 19.59 & 6.17 \\
\hline $\mathbf{4 f}$ & 1.34 & 2.33 & 19.59 & 5.02 \\
\hline $\mathbf{4 g}$ & 1.34 & 2.11 & 19.62 & 4.11 \\
\hline $\mathbf{4 h}$ & 1.01 & 2.96 & 24.26 & 7.11 \\
\hline $\mathbf{4 i}$ & 0.99 & 3.49 & 26.02 & 8.05 \\
\hline $\mathbf{4 j}$ & 1.03 & -0.55 & 11.88 & 4.91 \\
\hline $\mathbf{4 k}$ & 1.01 & -0.02 & 16.52 & 4.91 \\
\hline $\mathbf{4 m}$ & 1.37 & 1.46 & 14.49 & 4.29 \\
\hline \hline
\end{tabular}

* Fr values are calculated using $\mathrm{R}$. Rekker's equation (ref 20).

**MR and $\mathrm{L}$ are obtained from literature (refs. 21 and 22).
Table VI: Correlation matrix for the intercorrelation of various molecular descriptors.

\begin{tabular}{|c|c|c|c|c|}
\hline \hline \multirow{2}{*}{$\begin{array}{c}\operatorname{cog} \\
\text { MIC }\end{array}$} & $\begin{array}{c}\text { Log } \\
\text { MIC }\end{array}$ & Fr & MR & L \\
\cline { 2 - 5 } & 1.000 & & & \\
\hline Fr & -0.075 & 1.000 & & \\
\hline MR & -0.582 & 0.748 & 1.000 & \\
\hline L & -0.776 & 0.634 & 0.855 & 1.000 \\
\hline
\end{tabular}

For modeling antifungal activity against Scopulariopsis brevicaulis fungi, a stepwise regression analysis was carried out using maximum $\mathrm{R}^{2}$ method $^{23}$. The results obtained are presented in Table VII. A perusal of Table VII shows that only one parameter alone doesn't give good results, while the bi-parametic regression expression involving MR and $\mathrm{Fr}$ revealed statistically significant results. On the other hand, when MR is coupled with the Fr and $\mathrm{L}$, regression obtained was found superior than the bi-parametic model and the model resulted is shown below:

Equation

$\begin{aligned} \log \mathrm{MIC}= & 0.1272 \mathrm{Fr}-0.0101 \mathrm{MR}- \\ & 0.1333 \mathrm{~L}+1.8543\end{aligned}$

$\mathrm{n}=12$, S.E. $=0.0646, \mathrm{R}=0.955$,

$\mathrm{F}=27.56$ and $\mathrm{Q}=14.783$

Where, $\mathrm{n}$ is the number of compounds used, S.E. is the standard error of estimation, $\mathrm{R}$ is multiple correlation coefficient. F is F-statistics. From the model the $\mathrm{L}$ and Fr descriptors are the major factors for the exhibition of antifungal activity against 
Scopulariopsis brevicaulis fungi. i.e The bulkiness of the substituent has smaller factor for the activity. Also, the above equation shows that the coefficients of MR and $\mathrm{L}$ are negative while the coefficient of $\mathrm{Fr}$ is positive. That is the $\log$ MIC is negatively linearly correlated with $\mathrm{MR}$ and $\mathrm{L}$ and positively linearly correlated with Fr. In other words as the decrease in hydrophobicity and the increase in length of the substituent at N-5 position increase the antifungal activity against Scopulariopsis brevicaulis fungi. That compounds, with substituent at $\mathrm{N}-5$ position of 4C-6C length $(\mathbf{4 e}, \mathbf{4 h}, \mathbf{4 i})$ or contain hydrophilic carboxylic group $(\mathbf{4 j}, \mathbf{4 k})$, are most active (MIC values 9.67$10.85 \mu \mathrm{M} / \mathrm{ml})$.

Table VII: Regression parameters and quality of correlation for modeling log MIC ( $\mu \mathrm{mole} / \mathrm{ml})$ activity.

\begin{tabular}{|c|c|c|c|c|c|c|c|c|}
\hline $\begin{array}{c}\text { Model } \\
\text { No. }\end{array}$ & $\begin{array}{c}\text { Param. } \\
\text { Used }\end{array}$ & $\mathrm{Ai}=1,2,3$ & $\begin{array}{c}\text { B } \\
\text { intercept }\end{array}$ & S.E. & $\begin{array}{c}\text { Corr. } \\
\text { Coef. (R) }\end{array}$ & $\mathrm{R}^{2}$ & F-ratio & $\begin{array}{c}\mathrm{Q}= \\
\text { R/S.E. }\end{array}$ \\
\hline $1 \mathrm{a}$ & $\mathrm{Fr}$ & -0.0119 & 1.2418 & 0.1939 & 0.075 & 0.0056 & 0.06 & 0.386 \\
\hline $1 b$ & $\mathrm{~L}$ & -0.1018 & 1.7382 & 0.1226 & 0.776 & 0.602 & 15.13 & 6.329 \\
\hline $1 \mathrm{c}$ & MR & -0.0187 & 1.5307 & 0.1580 & 0.582 & 0.339 & 5.13 & 3.683 \\
\hline $2 a$ & $\begin{array}{l}\mathrm{Fr} \\
\mathrm{L}\end{array}$ & $\begin{array}{r}0.0597 \\
-0.1153 \\
\end{array}$ & 1.7285 & 0.1028 & 0.865 & 0.748 & 13.39 & 8.414 \\
\hline $2 b$ & $\begin{array}{c}\text { MR } \\
\mathrm{L}\end{array}$ & $\begin{array}{r}0.0097 \\
-0.1357\end{array}$ & 1.7500 & 0.1252 & 0.792 & 0.627 & 7.55 & 6.326 \\
\hline $2 c$ & $\begin{array}{c}\mathrm{Fr} \\
\mathrm{MR}\end{array}$ & $\begin{array}{r}0.0844 \\
-0.0271 \\
\end{array}$ & 1.5582 & 0.1320 & 0.765 & 0.585 & 6.34 & 5.795 \\
\hline 3 & $\begin{array}{c}\text { Fr } \\
\text { MR } \\
\mathrm{L}\end{array}$ & $\begin{array}{r}0.1272 \\
0.0101 \\
-0.1333\end{array}$ & 1.8543 & 0.0646 & 0.955 & 0.91 & 27.56 & 14.78 \\
\hline
\end{tabular}




\section{Conclusion}

In this work a series of 3-allyl-5substituted-2H-3,4,5,6-tetrahydro-1,3, 5 -thiadiazine-2-thione derivatives were synthesized and tested for antimicrobial activity. The results indicated that the antifungal activity against Scopulariopsis brevicaulis fungi could be successfully modeled by the combination of MR, Fr and L parameters. The QSAR model revealed that the decrease in hydrophobicity and the increase in length of the substituent at N-5 position increase the antifungal activity against Scopulariopsis brevicaulis fungi.

\section{REFERENCES}

1- Manfred E. Wolf "Burger's Medicinal Chemistry", $4^{\text {th }}$ Ed. John Wiley and Sons, New York, 1979, pp. 531-572.

2- M. Schorr, W. Durrcheimer, P. Klatt., G. Lommler, G. Nesemann and E. Schrinner, Arzneim-Forsch./Drug Res., 19, 1807 (1969).

3- M. Ertan, A. B. Tayhan and N. Yulug, Arch. Pharm., 323, 605 (1990).

4- A. El-Shorbagi, Arch. Pharm. Pharm. Med. Chem., 333, 281 (2000).

5- T. Aboul-Fadl and K. Hassanin, Pharmazie., 54, 244 (1999).

6- M. A. Hussein and A. ElShrobagi, A. Khallil, Arch. Pharm. Med. Chem., 334, 305 (2001).

7- M. Ertan, A. A. Bilgin, E. Palaska and N. Yulug, Arzneim-
Forsch./Drug Res., 42, 160 (1992).

8- J. Goksoyr, Acta Chemica Scandinavica, 18, 1341 (1964).

9- A. El-Shorbagi, Eur. J. Med. Chem., 29, 11 (1994).

10- A. Rieche, G. Hilgetag, A. Martini, O. Nejedly and J. Schegel, Arch Pharm., 293, 957 (1960).

11- A. Rieche, G. Hilgetag, A. Martini and R. Philippson., ibid, 294, 201 (1961).

12- U. Schmidt, E. Beyer and D. Martin., Dtsch. Akad. Wiss., 6, 458 (1964).

13- B. Rada, T. Hanusovska, L. Drobnica and P. Nemec, Acta Virol., 15, 329 (1971).

14- S. Sarac, M. Ertan and A. Balkan, Arch. Pharm. (Weinheim), 324, 449 (1991).

15- C. Ochoa, E. Perez, R. Perez, M. Suarez, E. Ochoa, H. Rodriguez, A. G. Barrio, S. Muelas, J. J. Nogal and R. A. Martinez, Arzneim.-Forsch./Drug Res., 49, 764 (1999).

16- T. Zsolnai., ibid., 21, 121 (1971).

17- W. Weuffen, D. Martin and W. Schade, Pharmazie, 18, 420 (1963).

18- S. T. Pai, M. W. Platt, Letters in Applied Microbiology, 20, 14 (1995).

19- C. Hansch and A. Leo, "Substituent Constants for Correlation Analysis in Chemistry and Biology" by C. Hansch and A. Leo, 1979, (Wiley and Sons), pp. 56-130. 
20- R. Rekker, Eur. J. Med. Chem. 14, 479 (1979).

21- Y. C. Martin, Quantitative Drug Design, A Critical Introduction in "Medicinal Research Series" (G.L. Grunewald, ed.), Marcel Dekker, Inc. New York and Basel, Vol. 8, 1978, pp. 61-110.
22- B. Skagerberg, D. Bonelli, S. Clementi, G. Cruciani and C. Ebert, Quant. Struct.-Act. Relat. 8, 32 (1989).

23- S. Chaterjee, A.S. Hadi, B. Price, Regression Analysis by Examples, $3^{\text {rd }}$ Ed., Wiley, New York (2000). 